\title{
Positron emission particle tracking: A powerful technique for flow studies
}

\author{
A. Buffler", K. Cole, T.W. Leadbeater and M.R. van Heerden \\ Department of Physics, University of Cape Town \\ Rondebosch, 7700, South Africa \\ *andy.buffler@uct.ac.za
}

Published 10 July 2018

\begin{abstract}
Positron emission particle tracking (PEPT) has developed into a flexible applied nuclear technique for measuring the trajectory of a single tracer particle moving in a system of granular or liquid flow or attached to a moving rigid body. The tracer particle is labelled with a radionuclide (such as ${ }^{18} \mathrm{~F}$ or ${ }^{68} \mathrm{Ga}$ ) that decays via positron emission. The nearly collinear $511 \mathrm{keV}$ annihilation gamma rays are detected in coincidence by a modified positron emission tomography (PET) camera, which defines their line of response (LOR). The chronologically measured LORs may then be used to triangulate the position of the moving tracer particle. We present an introduction to PEPT and illustrate the quality of measurements possible with a high-resolution PET scanner. Data are presented and discussed with reference to a few fundamental measurement scenarios and a framework for the metrology of PEPT is introduced.
\end{abstract}

Keywords: Positron emission particle tracking; flow visualization.

PACS numbers: $87.57 . u k, 47.80 . J k$

\section{Introduction}

Positron emission particle tracking (PEPT) has been developed into a useful technique ${ }^{1-6}$ for measuring the trajectory of a single tracer particle moving within a system of granular or liquid flow or attached to a moving rigid body. The tracer is labeled with a radionuclide that decays via positron emission, and the nearly collinear $511 \mathrm{keV}$ annihilation gamma rays are detected in coincidence by a modified positron emission tomography (PET) camera, defining their line of response (LOR). The chronologically measured LORs may be used to triangulate the position of the moving tracer. In principle, only two LORs are necessary, but the recording of non-useful LORs from the detection of gamma rays after undergoing Compton scattering between creation and detection, and the coincident detection of two gamma rays unassociated with the same annihilation event, means that a larger number of measured LORs is required. However, since hundreds of thousands of coincidence events can be processed by a PET camera every second, the tracking of particles moving as fast as $10 \mathrm{~m} / \mathrm{s}$ may be realized. Velocities and accelerations are determined from measured position-time coordinates

This is an Open Access article published by World Scientific Publishing Company. It is distributed under the terms of the Creative Commons Attribution 4.0 (CC-BY) License. Further distribution of this work is permitted, provided the original work is properly cited. 
$(x, y, z ; t)$ via an interpolation and differentiation algorithm. ${ }^{4,7,8}$ Other parameters, such as occupancy, residence time, mixing indices, diffusion constants, vorticity and porosity, are also routinely determined from the measured coordinates.

PEPT was formally postulated in the early 1990s by a group at the University of Birmingham. ${ }^{1-3}$ PEPT Cape Town ${ }^{9}$ became the second operational PEPT facility in the world in 2009, after the Positron Imaging Centre at the University of Birmingham. Over the last decade, a wide range of PEPT applications has been explored, both at the Positron Imaging Centre and PEPT Cape Town, in particular: powder mixing; ${ }^{10}$ particle and fluid behavior in granular beds; $;{ }^{112}$ fluidization phenomena; ${ }^{13,14}$ rotating drums; ${ }^{15,16}$ and minerals processing. ${ }^{17-19}$ As an example, PEPT measurements of a tracer tumbling inside a small rotating drum of internal diameter $7.0 \mathrm{~cm}$ and volume $1.0 \mathrm{~L}$, filled with glass beads of diameter $5.0 \mathrm{~mm}$ are shown in Fig. 1. The left panel shows a few thousand coordinates measured with a frequency of a few $\mathrm{kHz}$ axially projected onto the $x-y$ plane, and the right panel, the axially projected average velocity field determined for voxels of volume $2 \times 2 \times 2 \mathrm{~mm}^{3}$.
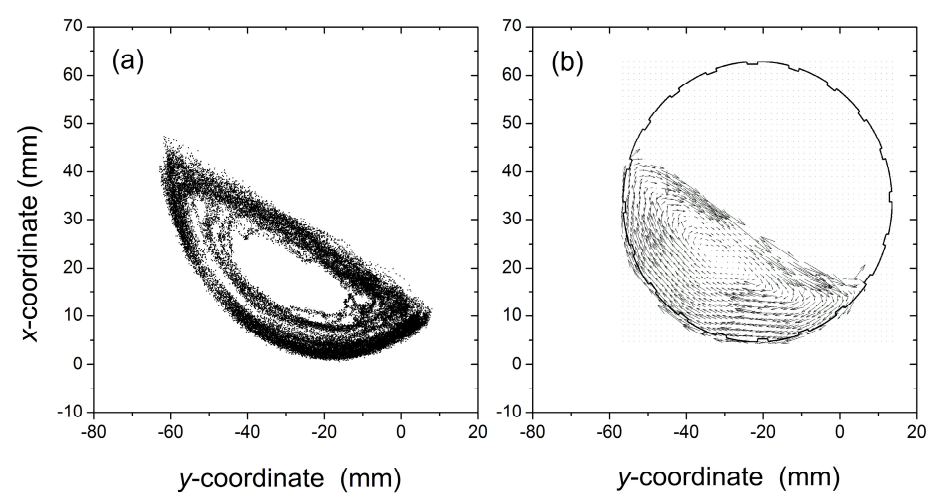

Fig. 1. An example of a PEPT measurement of (a) a few thousand locations ( $z$-axis projection) of a tracer moving within a small rotating drum filled with glass beads of diameter $5 \mathrm{~mm}$; and (b) the average velocity field determined for the tumbling tracer.

For PEPT studies, it is usually important for the shape and composition of the tracer to be like as possible to the material represented. Furthermore, the activity of the tracer must be such that sufficient LORs are recorded per second to produce a measurement of the trajectory of the tracer with a high location frequency and minimal uncertainty. There is often a tradeoff between tracer activity and physical characteristics. A large number of positron-emitting radioisotopes, with half-lives ranging from minutes to years, are in principle suitable for PEPT tracers. However, since it is frequently unrealistic to recover a tracer particle from the bulk of the liquid or powder under study, the half-life $\left(t_{1 / 2}\right)$ of the radioisotope should ideally be long enough to enable tracking over a practical experimental timescale, but short enough for the tracer to be discarded as radioactive waste after use. Radioisotopes commonly used for PEPT experiments are ${ }^{68} \mathrm{Ga}$ 
$\left(t_{1 / 2}=68 \mathrm{~min}\right)$ and ${ }^{18} \mathrm{~F}\left(t_{1 / 2}=109 \mathrm{~min}\right) .{ }^{22} \mathrm{Na}\left(t_{1 / 2}=2.6 \mathrm{y}\right)$ is often useful in experiments where the tracer can definitely be recovered, or for calibration.

A number of different techniques are used for the production of tracers. Ion-exchange techniques based on both weak-base and strong-base ionic exchange are used to label resin tracers with diameters less than $1000 \mu \mathrm{m}$ or so. ${ }^{20,21}$ Surface-modification techniques based on the introduction of particular metallic ions, e.g. $\mathrm{Fe}^{3+}$, onto the tracer surface often help enhance adsorption. It is sometimes necessary to seal the labeled tracer to prevent leaching of radioactive ions into the surrounding media. Tracers that include oxides may also be directly activated by producing ${ }^{18} \mathrm{~F}$ using an accelerator beam, ${ }^{22}$ which has the advantage that the tracer remains identical to the bulk from which the tracer stock is chosen. Larger tracers can be manufactured by micro-drilling into the particle, inserting the active material, and then backfilling and sealing the aperture. The activity with which a tracer particle may be labeled depends strongly on its size and composition. For typical PEPT applications, it has been found that tracer activity must be between 10 to $40 \mathrm{MBq}$ (about 300 to $1000 \mu \mathrm{Ci}$ ). In the absence of significant scattering and absorbing material in the system under study, the number of measured LORs per second is directly proportional to the activity with which the tracer is labeled. ${ }^{23}$ However, the system under study often presents a scattering environment that is dynamically changing with respect to the location of the tracer within the system, which can add complexity to the tracking analyses.

We present an introduction to PEPT and illustrate the quality of PEPT measurements possible with a high-resolution and high-efficiency PET scanner. Data are presented and discussed with reference to a few fundamental measurement scenarios and a framework for the metrology of PEPT is introduced.

\section{Experiments}

The laboratories of PEPT Cape Town ${ }^{9}$ are situated at iThemba LABS (Laboratory for Accelerator-Based Sciences) in Cape Town, South Africa, a national laboratory that operates a number of accelerators, including a $k=200$ separated-sector cyclotron. The cyclotron facility routinely produces light and heavy particle beams for nuclear physics research and radioisotope production. The PEPT Cape Town laboratories house the ECAT HR++ (Model: CTI/Siemens 966) PET camera (shown in Fig. 2) and a number of smaller PET systems. The HR++ was designed to achieve high resolution and high sensitivity for PET-based research. ${ }^{24}$ The camera consists of 48 rings of standard bismuth-germinate detector elements (each $4.39 \mathrm{~mm}$ transaxial $\times 4.05 \mathrm{~mm}$ axial $\times 30 \mathrm{~mm}$ deep, grouped in blocks of $8 \times 8$ ) with a ring diameter of $82 \mathrm{~cm}$, producing a 23.4-cm axial field of view. The data-acquisition system can maintain a maximum acquisition rate of about four million coincidence events per second, with a much higher sustained dataacquisition rate possible. ${ }^{24}$

For the present studies, a rotating disk apparatus (diameter $154 \mathrm{~mm}$ ) was positioned axially at the center of the field of view of the HR++ scanner, providing a stable platform onto which a tracer could be attached, and rotated using a DC motor. Point sources of 


\section{A. Buffler et al.}

${ }^{68} \mathrm{Ga}\left(t_{1 / 2}=68 \mathrm{~min}\right)$ were used as tracers for the studies. Each source was a Perspex cylinder of $10 \mathrm{~mm}$ diameter and $11.5 \mathrm{~mm}$ length, into which a small pyramid-shaped hole was drilled at the center. A drop of ${ }^{68} \mathrm{Ga}$ in solution was inserted in the hole with a pipette, allowed to evaporate, and sealed with cyanoacrylate polymer (superglue). The active volume of each source was less than $1 \mathrm{~mm}^{3}$, and their initial activities ranged from 10 to $740 \mathrm{kBq}$, measured with a calibrated Capintec CRC-25R ionization chamber. PEPT runs were undertaken as a function of a variety of these three parameters: position on the disk; rotation speed of the disk; and tracer activity.

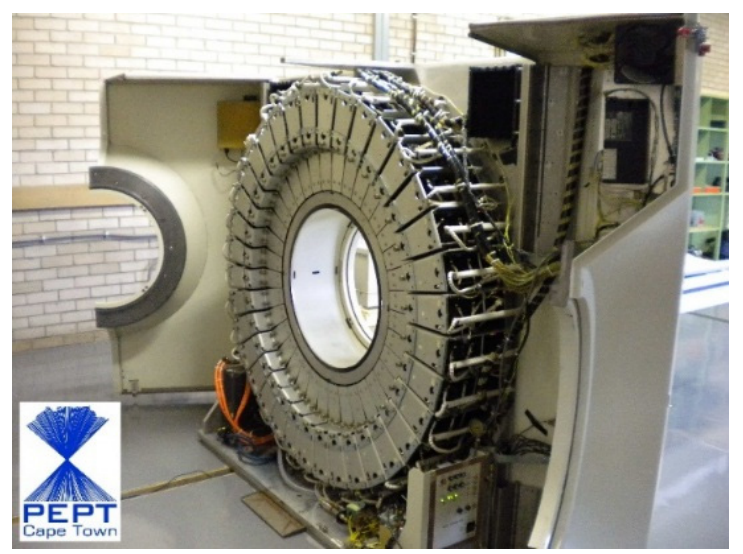

Fig. 2. The Siemens HR++ PET scanner at PEPT Cape Town, which has been adapted for PEPT.

Data are recorded chronologically, where each coincidence event is written to a circular buffer describing the two detector elements in the ring that give rise to each LOR and a timestamp accurate to one millisecond per event. Each data word representing a single LOR contains 32 bits of data, and circular buffers are recorded to disk every $10^{4}$ events. To represent the LORs in software, each event is deconstructed into Cartesian coordinates describing position and time for each LOR. The analysis of list-mode data from a PEPT run begins with slicing a series of chronological coincidence events (recorded LORs) into equal groups of events $N$. The algorithm for determining the location of the tracer is based on the premise that for a given $N$, all uncorrupted LORs should ideally intersect at a single point in space, i.e. the location of the tracer. In practice, LORs typically do not intersect, for a variety of reasons; thus, the approach is to find the point in space that minimizes the sum of distances to the LORs. Since a proportion of the LORs are corrupted by one or both of the coincident gamma rays undergoing Compton scattering before detection, or by the detection of uncorrelated gamma rays in coincidence, the algorithm must disregard these events and determine the location from a set of uncorrupted LORs. The current implementation of the algorithm requires the user to specify the set size $N$, the percentage $f$ of $N$ LORs to use in the triangulation of each location, and a maximum acceptable uncertainty for the final 
solution. ${ }^{1}$ The choice of $N$ and $f$ depend of the total LORs recorded in the run and the proportion that have been corrupted. Details of the algorithm have been reported elsewhere. ${ }^{1,6,7}$

As an illustration, Fig. 3 shows an axial projection of about 1000 LORs measured in about one second by the HR++ scanner, before and after application of the triangulation algorithm. It is expected that the number of events undergoing Compton scattering will depend on the amount and nature of the material in the system under study. However, since the $\mathrm{HR}++$ scanner only records events for which the LORs are associated with either the same or a neighboring ring of detector elements, events from Comptonscattered gamma rays are largely eliminated.
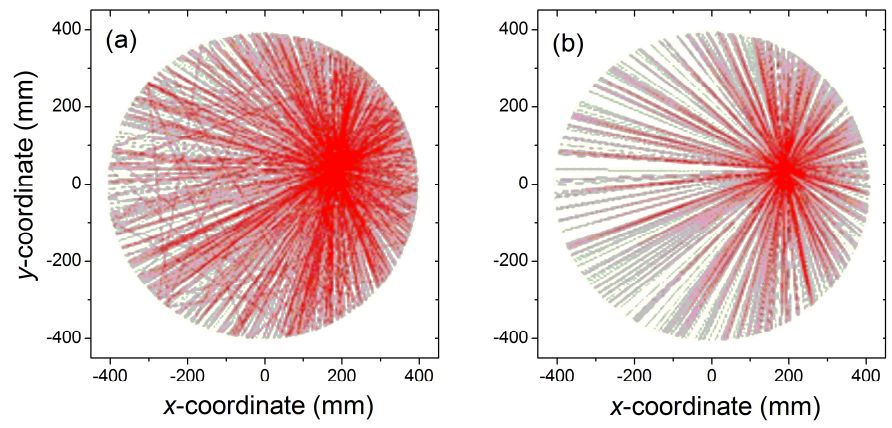

Fig. 3. An axial projection of one thousand lines of response measured in about one second by the Siemens HR++ PET scanner before (left) and after (right) application of the PEPT algorithm.

\section{Analysis and Results}

\subsection{Stationary tracer}

We discuss data measured for a stationary tracer labelled with $1000 \mathrm{kBq}$ of ${ }^{68} \mathrm{Ga}$ placed near the center of the field of view of the HR++ scanner. The PEPT triangulation algorithm was applied over a range of $N$ and $f$, and a statistical measure of the relative uncertainty in the $i^{\text {th }}$ measured location $\left(x_{i}, y_{i}, z_{i}\right)$ was calculated using

$$
u(x)=\sqrt{\frac{\sum\left(x_{i}-\bar{x}\right)^{2}}{n}} ; u(y)=\sqrt{\frac{\sum\left(y_{i}-\bar{y}\right)^{2}}{n}} \text { and } u(z)=\sqrt{\frac{\sum\left(z_{i}-\bar{z}\right)^{2}}{n}}
$$

where $n$ is the number of measured locations (1000 in this case) and $\bar{x}, \bar{y}$ and $\bar{z}$ are the mean values. The results in Fig. 4 indicate that a minimum in $u$ occurs between $f=70 \%$ and $f=80 \%$ for each of $x, y$, and $z$.

Figures $5(\mathrm{a}-\mathrm{c})$ presents locations $(x, y, z)$ measured for the stationary tracer for 700 ms of the run. The data were analyzed using $f=80 \%$ and $N=400$ (see Fig. 4), chosen as a compromise between maximizing location rate and minimizing uncertainty. Figure $5(\mathrm{~d}-$ f) shows the frequency distributions of these data. Half the FWHMs of these 


\section{A. Buffler et al.}

distributions, determined by fitting a Gaussian to each histogram, are a measure of the minimum uncertainty in the locations measured by the $\mathrm{HR}++$ scanner, using ${ }^{68} \mathrm{Ga}$ as the labeling isotope. These were determined to be approximately $0.7 \mathrm{~mm}$ in each case.
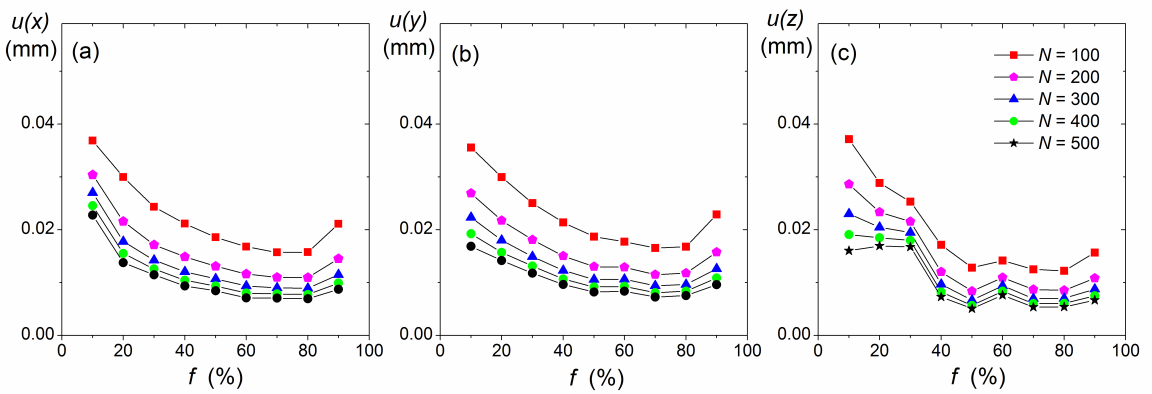

Fig. 4. Relative statistical uncertainties $u$ in the coordinates (a) $x$, (b) $y$, and (c) $z$, measured via PEPT for a stationary tracer positioned near the center of the HR++ scanner's field of view, as a function of both $f$ and $N$. The lines are to guide the eye.
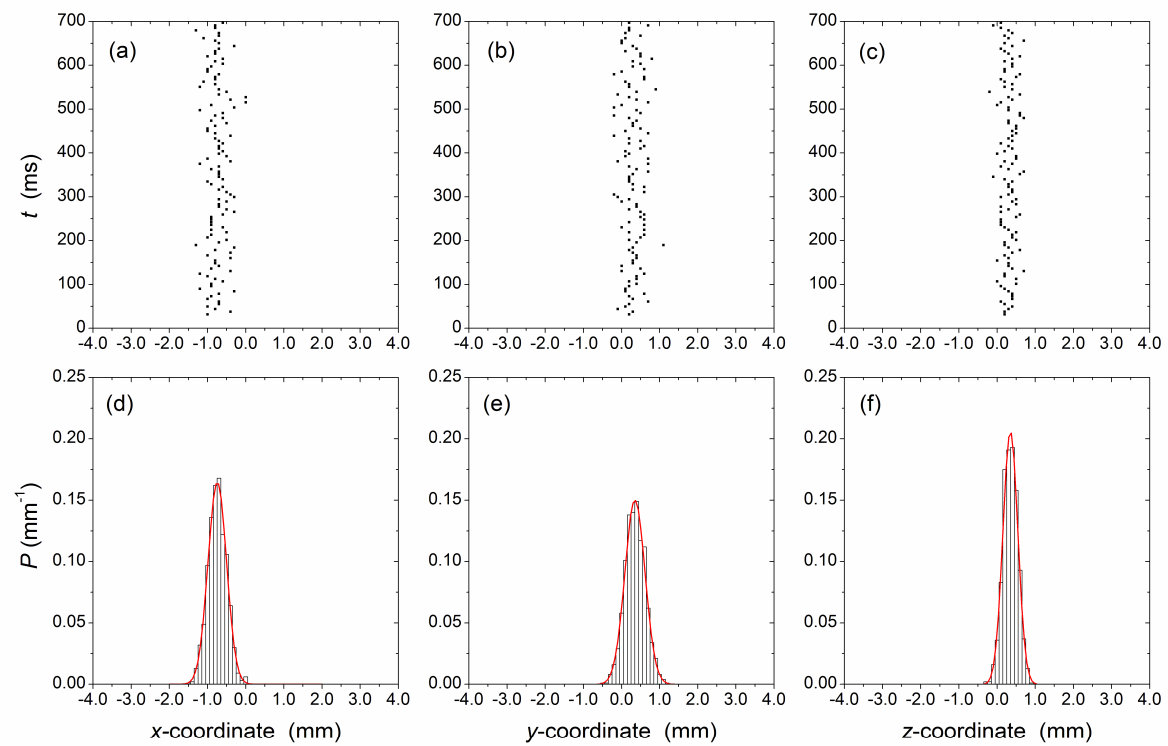

Fig. 5. Coordinates (a) $x$, (b) $y$, and (c) $z$, measured using $f=80 \%, N=400$, for a short period (700 ms) for a small stationary tracer near the center of the field of view of the HR++ scanner; and $(\mathrm{d}-\mathrm{f})$ frequency distributions of these coordinates, fitted with Gaussian functions (red).

\subsection{Rotating tracer}

Data are presented for a ${ }^{68} \mathrm{Ga}$ tracer (activity $47 \mathrm{kBq}$ ) attached to a rotating platform (106 rpm, radius $0.077 \mathrm{~m}$, tangential speed $0.856 \mathrm{~m} / \mathrm{s}$ and centripetal acceleration 
$9.52 \mathrm{~m} / \mathrm{s}^{2}$ ), positioned axially in the center of the field of view of the HR++ scanner. Figure 6 shows measured (a) $x$, (b) $y$ and (c) $z$ coordinates for the rotating tracer for 5000 ms of a 30-second run (total events 1600), using $N=400$ and $f=70 \%$. Figure 7 shows plots of (a) all measured $x-y$ coordinates, (b) the average velocity field, and (c) the average acceleration field in the azimuthal $(x-y)$ plane. The $x-y$ projected velocities and accelerations have been averaged in a $32 \times 32$ grid with each square pixel element covering an area of $5 \times 5 \mathrm{~mm}^{2}$.
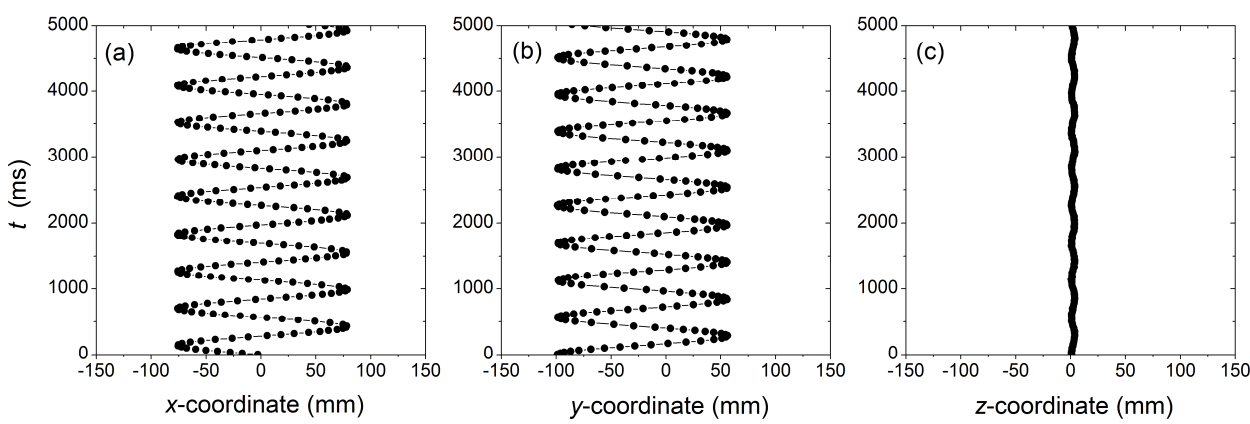

Fig. 6. Coordinates (a) $x$, (b) $y$, and (c) $z$, measured for a short period (5000 ms) for a small tracer rotating at $106 \mathrm{rpm}$ in the $x-y$ plane near the center of the HR++ scanner's field of view
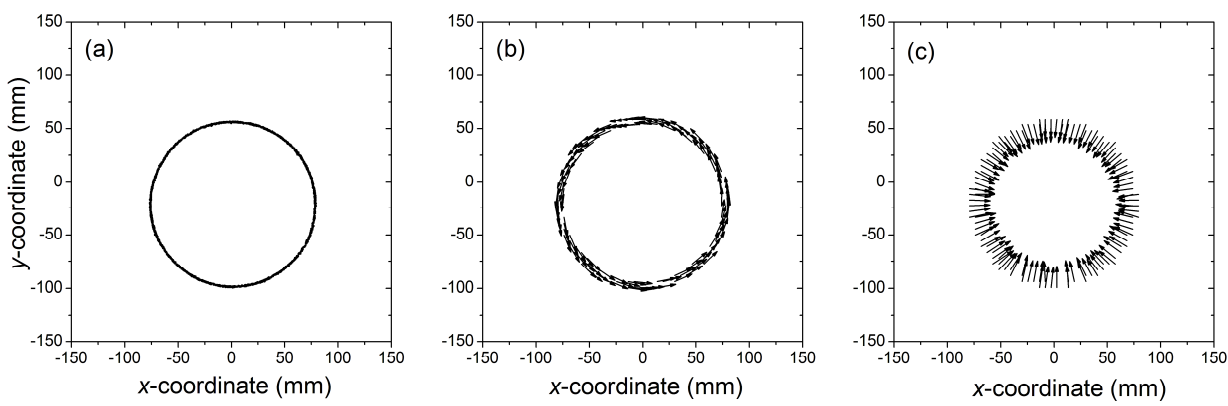

Fig. 7. (a) All 1600 ( $x$, y) locations measured for the rotating tracer (Fig. 6), and $x-y$ projections of the (b) average velocity field and (c) average acceleration field, binned in a grid of $5 \times 5 \mathrm{~mm}^{2}$. The vectors in (b) and (c) are arbitrarily scaled.

A measure of the statistical uncertainty $u\left(r_{x y}\right)$ in the measured positions was defined as

$$
u\left(r_{x y}\right)=\sqrt{\frac{\sum\left(\Delta r_{i}\right)^{2}}{n}},
$$

where the $\Delta r_{i}$ are the perpendicular distances between each $(x, y)$ coordinate measured via PEPT and the expected radial position of the tracer, given by a fully $3 \mathrm{D}$ least-squares fit of a circle to the entire set of $n=1600$ measured locations. The $z$-coordinates were 


\section{A. Buffler et al.}

excluded from this analysis, and it is noted that the slight deviation from coaxiality seen in Fig. 7(c) will contribute additional uncertainty. The PEPT triangulation algorithm was applied over a range of $N$ and $f$, and $u\left(r_{x y}\right)$ was calculated for each combination. The values of $u\left(r_{x y}\right)$ determined in this way are presented in Fig. 8(a). It can be seen that a minimum in $u\left(r_{x y}\right)$ occurs between $f=60 \%$ and $f=70 \%$, and that $N=400$ provides a reasonable compromise between minimizing uncertainty and maximizing location frequency.
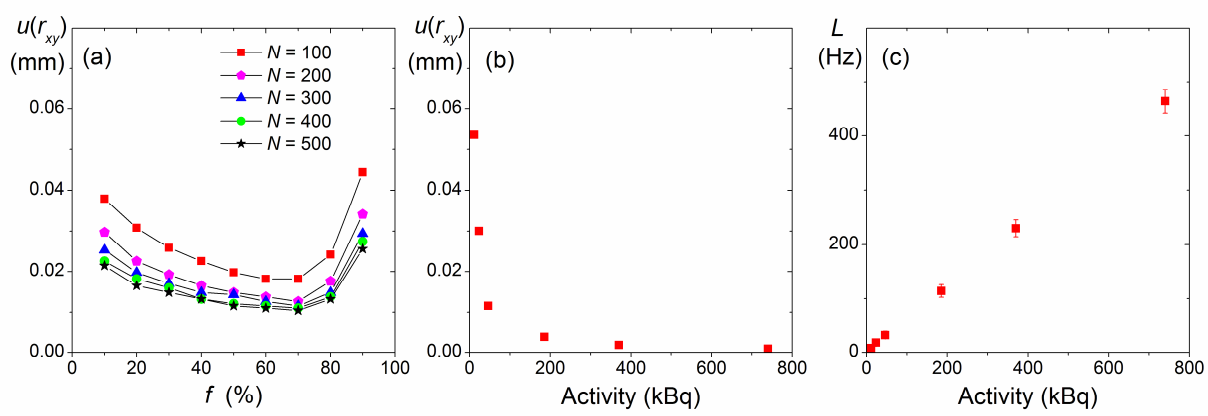

Fig. 8. (a) Uncertainties $u\left(r_{x y}\right)$ in the measured positions of a rotating tracer as a function of both $f$ and $N$. The lines are to guide the eye. (b) Uncertainties $u\left(r_{x y}\right)$ measured for the rotating tracer, a function of tracer activity using $f=70 \%, N=400$. (c) Location rate $L$ measured for the rotating tracer as a function of tracer activity, using $f=70 \%, N=400$, which is linear, as expected for this range of activity.

\subsection{Tracer activity}

The same analyses applied to the rotating tracer were completed for a range of tracer activities between $10 \mathrm{kBq}$ and $740 \mathrm{kBq}$. Figure 8 also shows (b) $u\left(r_{x y}\right)$ measured as a function of tracer activity using $f=70 \%$ and $N=400$, and (c) the location rate $L$ measured under the same conditions. As expected, the statistical uncertainty $u\left(r_{x y}\right)$ deceases asymptotically to a minimum value as tracer activity increases, while location rate $L$ increases linearly with activity.

\section{Discussion}

An introduction to the metrology of PEPT has been presented with reference to data measured using the Siemens HR++ scanner at PEPT Cape Town. The high number of individual detector elements $(27,648)$ in the $\mathrm{HR}++$ scanner results in a high positional resolution of the measured LORs, and the fast coincidence logic can sustain a high data rate. Together these provide unique opportunities for PEPT measurements over a wide range of tracer activities and flow environments. The HR++ scanner records lines of response in strict chronological order of detection, but is limited by a time resolution of a millisecond in the recorded list-mode data file. With respect to measured $(x, y, z ; t)$ coordinates, based upon data obtained from a wide array of measurement scenarios using 
the $\mathrm{HR}++$ scanner, the minimum uncertainty associated with a single measured location is on the order of a millimeter. ${ }^{25}$

The present data offers insight into the multiple components that contribute to a PEPT measurement. A full uncertainty budget for PEPT with the HR++ scanner is yet to be realized and will require deeper understanding of the physics limitations of PEPT. Such a budget will include uncertainty contributions from the detection system, the activity of the tracer, the species of radionuclide used, the nature of the material between tracer and detector, the speed of the tracer, and the triangulation algorithm. Increased scattering material in the system reduces the recorded coincidence rate, which translates directly into the number of measured LORs available for triangulations. This may be easily compensated for by increasing the activity of the tracer particle. However, increased activity does not reduce the uncertainty below a minimum value for the conditions of the run. ${ }^{25}$ Consequently, analysis of the measured LORs may benefit from context-based optimization. Monte Carlo simulations of gamma-ray transport in the system may be useful in this regard.

PEPT is now an established nuclear technique that has proven useful for measuring flow parameters in complex engineering systems. Work at PEPT Cape Town will continue to focus on a wide range of granular and liquid systems and application of PEPT in new contexts. For example, we are developing a modular PEPT system for use in the field $^{26}$ and new digital data-acquisition systems. ${ }^{27,28}$ The most important advances, however, are likely to be made with respect to tracer design. Plans are underway to develop a capability to directly activate tracers using cyclotron beams and produce ${ }^{11} \mathrm{C}$ for gas-based studies that combine PEPT with traditional PET imaging. For PEPT to be reliably applicable to liquid and gaseous systems such as flotation cells, the state of the art needs to be extended to the routine production of tracers smaller than 30 microns. $^{29}$

\section{References}

1. D.J. Parker, C.J. Broadbent, P. Fowles, M.R. Hawkesworth and P.A. McNeil, Nucl. Instr. Meth. A326, 592 (1993).

2. D.J. Parker, R.N. Forster, P. Fowles, and P.S. Takhar, Nucl. Instr. Meth. A477, 540 (2002).

3. J.P.K. Seville, A. Ingram and D.J. Parker, Chem. Eng. Res. Des., 83, 788 (2005).

4. S. Bakalis, P. W. Cox, A. B. Russell, D.J. Parker and P.J. Fryer, Chem. Eng. Sci. 61, 1864 (2006).

5. D.J. Parker, T.W. Leadbeater, X. Fan, M.N. Hausard, A. Ingram and Z. Yang, Meas. Sci. Tech. 19 (2008).

6. T.W. Leadbeater, D.J. Parker and J. Gargiuli, Particuology 10, 146 (2012).

7. D. Boucher, Z. Deng, T. Leadbeater, R. Langlois, M. Renaud and K.E. Waters, Min. Eng. 62, 120 (2014).

8. A. Guida, A.W. Nienow and M. Barigou, AIChE Journal 58, 31 (2012).

9. A. Buffler, I. Govender, J.J. Cilliers, D.J. Parker, J-P. Franzidis, A. Mainza, R.T. Newman, M. Powell and A. van der Westhuizen, PEPT Cape Town: a new positron emission particle tracking facility at iThemba LABS, in Proc.2009 International Topical Meeting on Nuclear Research Applications and Utilization of Accelerators (IAEA, Vienna, 2010), STI/PUB/1433, ISBN 978-92-0-150410-4, ISSN 1991-2374, CD-ROM.

10. M. Bickell, A. Buffler, I. Govender and D.J. Parker, Nucl. Instr. Meth. A682, 36 (2012). 


\section{A. Buffler et al.}

11. T.W. Martin, J.P.K. Seville and D.J. Parker, Chem. Eng. Sci. 62, 3419, (2007).

12. R.D. Wildman, C.M. Hrenya, J.M. Huntley, T.W. Leadbeater and D.J. Parker, Granular Matter 14, 215 (2012).

13. C.R.K. Windows-Yule, T. Weinhart, D.J. Parker and A.R. Thornton, Phys. Rev. Lett. 112, 23 (2014).

14. M.S. van Buijtenen, W.J. van Dijk, N.G. Deen, J.A.M. Kuipers, T.W. Leadbeater and D.J. Parker, Chem. Eng. Sci. 66, 2368 (2011).

15. P. Garcia-Trinanes, J. Seville, B. Boissière, R. Ansart, T.W. Leadbeater and D.J. Parker, Chem. Eng. Sci. 146, 346 (2016).

16. D.J. Parker, A.E. Dijkstra, T.W. Martin and J.P.K. Seville, Chem. Eng. Sci. 52, 2011 (1997).

17. A.J. Morrison, I. Govender, A.N. Mainza and D.J. Parker, Chem. Eng. Sci. 152, 186 (2016).

18. K. Cole, K.E. Waters, D.J. Parker, S.J. Neethling and J.J. Cilliers, Chem. Eng. Sci. 65, 1887 (2010).

19. J. Chaouki, F. Larachi and M.P. Dudukovic, Ind. Eng. Chem. Res. 36, 4476 (1997).

20. K. Cole, A. Buffler, J.J. Cilliers, I. Govender, J.Y.Y. Heng, C. Liu, D.J. Parker, U.V. Shah, M. van Heerden and X. Fan, Powder Tech. 124, 18 (2014).

21. X. Fan, D.J. Parker and M.D. Smith, Nucl. Inst. Meth. A562, 345 (2006).

22. D. Boucher, A. Jordens, J. Sovechles, R. Langlois, T.W. Leadbeater, N.A. Rowson, J.J. Cilliers and K.E. Waters, Min. Eng. 100, 155 (2017).

23. T.J. Spinks, T. Jones, P.M. Bloomfield, D.L. Bailey, M. Miller, D. Hogg, W.F. Jones, K. Vaigneur, J. Reed, J. Young, D. Newport, C. Moyers, M.E. Casey and R. Nutt, Phys. Med. Biol. 45, 2601 (2000).

24. D.J. Parker and T.W. Leadbeater, New life in old PETs - The work of the University of Birmingham Positron Imaging Centre, in 6th International Symposium on Process Tomography, Cape Town (2012).

25. T.S. Volkwyn, A. Buffler, I. Govender, J-P. Franzidis, A.J. Morrison, A. Odo, N.P. van der Meulen and C. Vermeulen, Min. Eng. 24, 261 (2011).

26. D.J. Parker, T.W. Leadbeater, X. Fan, M.N. Hausard, A. Ingram and Z. Yang, Nucl. Instr. Meth. A604, 339 (2009).

27. T.W. Leadbeater and D.J. Parker, Nucl. Instr. Meth. A604, 355 (2009).

28. W.F. Jones, M.E. Casey, L.G. Byars, and S.G. Burgiss, Nucl. Instr. Meth. A326, 592 (1993).

29. K. Cole, A. Buffler, N.P. van der Meulen, J.J. Cilliers, J.-P. Franzidis, I. Govender, C. Liu and M.R. van Heerden, Chem. Eng. Sci. 75, 235 (2012). 